\title{
Robotic Bronchoscopy
}

\author{
Klamkin $\mathbf{M}^{1}$ and Kazachkov $\mathbf{M}^{2}$
}

${ }^{1}$ Marine Advanced Technology Education Center Robotic Program, Canada

${ }^{2}$ Department of Pediatics, Hassenfeld Children's Hospital, NYU Langone Health, New York, USA

\begin{abstract}
Despite major advances in robotic surgery, which occurred during last 25 years, robotic bronchoscopy remains to be a new and exciting field. This is a short review of history, development and potential implications of robotic bronchoscopy.
\end{abstract}

Keywords: Robot bronchoscopy; Robotic surgery; Gastrointestinal endoscopy

\section{Introduction}

Robot-assisted surgery was started as an attempt to improve minimally invasive surgical techniques and to lessen the size of surgical incision. The first surgical robot (Arthrobot by McEwen, Auchinleck and Day) was developed in 1983 in Vancouver [1] and was used in assisting with orthopedic surgical procedures. At the same time the US State Department of Defense started development of Green SRI telepresence surgical system to assist the surgeon at the battlefield. Shortly after that, initial experiments with a six-axis unimate (PUMA) were started and resulted in successful performance of urethral resection of the prostate at Imperial College, London in 1988 [2]. In late 1990s, the first 200 laparoscopic surgeries with the system created by Intuitive Surgical were completed [3] leading to Food and Drug Administration (FDA) approval of this robotic system (Da Vinci Surgical System) in 2000. The Da Vinci nowadays is used to perform a multitude of operations, including but not limited to hysterectomies, nephrectomies, radical prostatectomies, and pancreatectomies. It uses a rigid rod design with manipulators or tools attached to the end of the rod and controlled by the surgeon [4].

\section{Development of Bronchoscopy Robot}

The main principals of robotic endoscopies were mastered in the process of development of robots for gastrointestinal endoscopy. Several ready-made systems exist, including the robotic steering and automated lumen centralization system (RS-ALC), which simply attaches an endoscope to control wheels, the endoscopic operation robot (EOR), which allows the surgeon to move the endoscope forward, backward, up, down, left, right, and rotate. More complex systems include the Neoguide by Intuitive Surgical, which is designed to minimize unintentional lateral forces by conforming to the natural shape of the colon, and the Aeroscope and Endotics, which use balloon systems to overcome the same problem, creating a seal with the colonic wall, using the pressure differential to move the endoscope. Some systems also include traction cable actuation for tooling on the end of the endoscope. All of these systems accomplish one common goal- assisting the surgeon in performing gastrointestinal endoscopies, whether through simply delivering the endoscope to the desired location, or provide surgeons with access to surgical tools on the end of the endoscope [5].

Despite all advances in robotic GI endoscopy, robotic bronchoscopy is very young and new field. Auris Surgical Robotics has been a pioneer in robotic bronchoscopy. Its first robotic bronchoscopic system (ARES) was approved by FDA in 2016. In order to minimize training times and facilitate convenience of usage, simple control systems, that can still take advantage of all the technical features of the system, were developed. Thus, the ARES robotic bronchoscopy system utilizes a familiar joystick style control system, where the surgeon moves joysticks, which translate to movements of tools in the patient. This system is completely user-controlled, with no machine assistance in the control of the tools, or tactile feedback in the joystick. In addition to the joystick system, the system also features a touch screen and several buttons to activate special functions such as the "pause" feature, which fixes the bronchoscope in place. The robotic bronchoscope system has sheath diameter of $5.7 \mathrm{~mm}$ with bronchoscope diameter $4.4 \mathrm{~mm}$ and working channel of $2.1 \mathrm{~mm}$ [6].

\section{Practical Implications}

First practical experience with the Auris robotic endoscopic system was recently reported by Chen et al. [6]. The investigators compared the ability of access into the bronchial tree by $4.4 \mathrm{~mm}$ conventional thin bronchoscope and robotic endoscopic system (RES). Eight segmental bronchi were examined in five cadavers. Overall, RES accessed on average $9.6(\mathrm{SD}=2.3)$ generations compared to $6.6(\mathrm{SD}=2)$ generations using the conventional thin bronchoscope $(\mathrm{p}=0.0001)$. In all airways, RES was advanced beyond the conventional bronchoscope by a minimum of 2 generations (Range 2-6). Insertion depth from the main carina demonstrated an overall average distance of $172 \mathrm{~mm}$ $(\mathrm{SD}=43.8)$ using the RES compared with $128.0 \mathrm{~mm}(\mathrm{SD}=27.5)$, with the bronchoscope $(\mathrm{p}=0.0009)$. The authors concluded that the RES accessed deeper into the lung periphery of human cadaveric models compared with a similarly sized conventional thin bronchoscope. They also noticed that the outer sheath design supporting the telescoping endoscope may provide added stability and column strength when attempting to advance further into peripheral airways. They suggested that the potential clinical implications of additional reach into the lung periphery may translate into improved diagnostic yields for peripheral lesion biopsy via an endoscopic approach.

Recently Rojas-Solano et al. [7] published case series of practical use of RES in 15 adult patients with "bronchus sign", which was defined as the presence of a bronchus leading to or contained within the target nodule as seen in a high-resolution chest CT scan. Of note,

*Corresponding author: Mikhail Kazachkov, Department of Pediatics, Hassenfeld Children's Hospital, NYU Langone Health, New York, USA, Tel: 646-501-0536; E-mail: Mikhail.Kazachkov@nyumc.org

Received August 02, 2018; Accepted August 16, 2018; Published August 23 2018

Citation: Klamkin M, Kazachkov M (2018) Robotic Bronchoscopy. J Pulm Respir Med 8: 466. doi: 10.4172/2161-105X.1000466

Copyright: $\odot 2018$ Klamkin M, et al. This is an open-access article distributed under the terms of the Creative Commons Attribution License, which permits unrestricted use, distribution, and reproduction in any medium, provided the original author and source are credited. 
the bronchoscope used in the RES was smaller $(3.2 \mathrm{~mm}$ with $1.2 \mathrm{~mm}$ working channel) than original. CT scan and fluoroscopy were used to aid the approach of the bronchoscope to the target lesion. No major complications were reported. Tissue acquisition under direct visualization was performed in 14 patients (93\%). Malignant lesions were identified in 9 patients and benign in 5. Only one patient required additional surgical procedure for non-diagnostic biopsy. Despite the fact that there was no comparison with biopsies done with conventional bronchoscope, the investigators were encouraged by the control and stability of the robotic bronchoscope and instruments in the periphery as well as by the reach achieved.

It is possible that certain features of the robotic bronchoscopic system, such as circular articulation as well as advanced precision in placement in the airway may become very attractive to interventional pulmonologists. As the above reviewed studies shows, it may find major applications in diagnostic pulmonary oncology and, in general, in facilitating of biopsies of nodular pulmonary lesions. Pediatric bronchologists may get interested in potential implications of this system for removal of foreign bodies of the airway taking into consideration its ability to "lock" the bronchoscope in the desired location [8], which gives the operator the ability to introduce a specific tool to the foreign object. Obviously, the large diameter of the sheath becomes an obstacle to this particular pediatric application at present.
In summary, robotic bronchoscopic system is being developed. It may have certain technical features and practical implications, which make it attractive to practitioners. However, prospective clinical trials may be needed to establish the safety and effectiveness of the robotic bronchoscopy.

\section{References}

1. Mohammad S (2013) Robotic surgery. J Oral Biol Craniofac Res 3: 2.

2. Davies BL, Hibberd RD, Coptcoat MJ, Wickham JE (1989) A surgeon robot prostatectomy- a laboratory evaluation. J Med Eng Technol 13: 273-273.

3. Himpens J, Leman G, Cadiere GB (1998) Telesurgical laparoscopic cholecystectomy. Surg Endosc 12: 1091.

4. Satava RM (2002) Surgical robotics: The early chronicles: a personal historical perspective. Surg Laparosc Endosc Percutan Tech 12: 6-16.

5. Yeung BP, Chiu PW (2016) Application of robotics in gastrointestinal endoscopy: A review. World J Gastroenterol 22: 1811-1825.

6. Chen A, Wahidi M, Gillespie C, Cicenia J, Machuzak M, et al. (2017) Robotic endoscopic airway challenge: REACH assessment. Chest 152: A882.

7. Rojas-Solano JR, Ugalde-Gamboa L, Machuzak M (2018) Robotic bronchoscopy for diagnosis of suspected lung cancer: A feasibility study. J Bronchology Interv Pulmonol 25: 168-175.

8. Don Tanaka (2018) Personal communication. 